\title{
Mechanical Design and Kinematic Simulation of Automated Assembly System for Relay
}

\author{
Zhenghao Liu, Hong Chen* \\ College of Mechatronics and Control Engineering, Shenzhen University, Shenzhen, China \\ Email: 2141160410@email.szu.edu.cn, chenhong@szu.edu.cn
}

Received 14 October 2015; accepted 11 January 2016; published 14 January 2016

Copyright (C) 2016 by authors and Scientific Research Publishing Inc.

This work is licensed under the Creative Commons Attribution International License (CC BY). http://creativecommons.org/licenses/by/4.0/

c) (i) Open Access

\begin{abstract}
By means of Solid Works, three-dimensional model of automated assembly system was established, and kinematic simulation based on Solid Works Motion of assembly process for relay was performed. The simulation results proved the feasibility of mechanical design. Eventually, the productivity was estimated based on simulation analysis. The mechanical design provided a solution with high reference value to practical design of automated assembly system for relay.
\end{abstract}

\section{Keywords}

\section{Relay, Mechanical Design, Solid Works, Kinematic Simulation, Solid Works Motion}

\section{Introduction}

In modern product manufacturing, automated assembly technology has been one of the key technologies [1]. The automated assembly technology is a comprehensive technology which combines the mechanical design, robotics and sensor and detection techniques [2]. As an important part for industrial production, the automated assembly can decide the total cost of production and productivity. And with the increasing functional demand for industrial products, more advanced automated assembly technology has access to meet the sophisticated demand [3]. Therefore, the automated assembly technology has become a hotspot of the current research. However, it exists some disadvantages of the automated assembly technology, such as inefficiency, high cost and complex mechanical structure [4] [5].

There are seldom study work on mechanical design and kinematic simulation of the automated assembly technology in the current studies. An automated assembly system is studied in this paper. The system is de-

\footnotetext{
"Corresponding author.
} 
signed for assembling a kind of relay by the machinery. Based on the mechanical structure of the assembly system, the whole working process of assembling the relay is achieved.

This paper is organized as follows. Principles of automated assembly system for relay are clarified in Section 2; the mechanical structure is illustrated as well. Section 3 focuses on the mechanical design of the Yoke, Bobbin and Base assembly part, including feed design, location design, clamping design and assembly design for the Yoke, Bobbin and Base. Kinematic simulation results and productivity estimation are shown in Section 4. Finally, conclusions are presented in Section 5.

\section{Principles of Automated Assembly System for Relay}

The main purpose of this project is to design a new practical automated assembly system for relay to meet work requirements of enterprises production. The working process of the assembly system is showing in Figure 1.

The procedure for each component involves eight steps and can be described as follows. At first, Bobbin is inserted into Yoke. The assembly of Yoke \& Bobbin inserts into Base together in the next step. Thirdly, Hinge embeds in semi-manufactures. And Armature embeds in semi-manufactures in the fourth step. In the fifth step, SCC inserts into semi-manufactures. B-T and M-T insert into semi-manufactures in the sixth step. Then card covers semi-manufactures in the seventh step. Finally, case covers semi-manufactures. Since then, an intact relay is finishing assembling.

\section{Mechanical Design of Automated Assembly System for Relay}

\subsection{Mainly Part of Automated Assembly System for Relay}

To facilitate the mechanical design of the automated assembly system for relay, the working process of assembly system can be mainly divided into three parts, the Yoke, Bobbin and Base assembly part, the Hinge and Armature assembly part and the SCC, B-T and M-T assembly part, which can be shown in Figure 2.

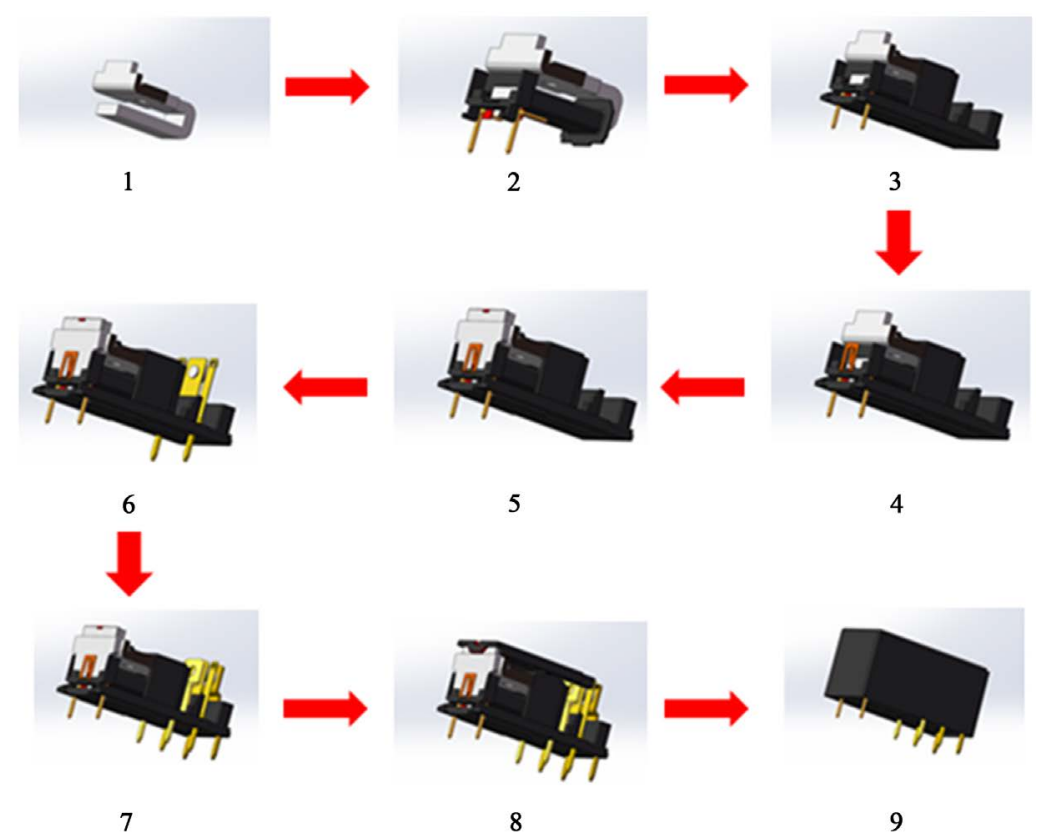

Figure 1. The working process of automated assembly system for relay.

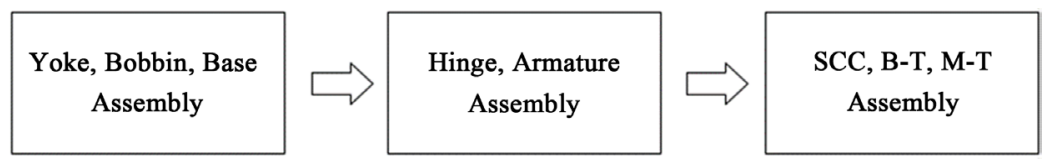

Figure 2. The mainly part of automated assembly system for relay. 
In this paper, we choose the mechanical design of Yoke, Bobbin and Base assembly part to analysis. Figure 3 shows the overall mechanical design of Yoke, Bobbin and Base assembly part. This part consists of eight customized sub-structures. Each sub-structure's design aims at different shapes of components made up the relay, and necessary mechanical actions realized the assembly function.

\subsection{Yoke, Bobbin and Base Assembly Part Design}

1) Mechanical design of feed, location and clamping for Yoke

It's unnecessary to protect the surface and structure of Yoke in particular, so the combination of vibration tray and linear feeder is chosen to be the means for transporting Yoke. And Yokes in the linear slider stand closely in a row so that are inconvenient to capture by the manipulator. The structure of location for Yoke is designed to separate the Yoke in the head of the linear slider and the Yoke in the next, then the manipulator can clamp the Yoke easily. The Air TACHF Series Gripper [6] is effective and stable for clamping as a clamping component.

2) Mechanical design of feed and clamping for Bobbin

Compared with the feed of Yoke and Base, Bobbins surrounded by very thin copper wires get damage easily in transmission for assembly in the next step. A specialized pallet with $12 \times 12$ rooms for storing Bobbins is designed as a feeder. Because of the discontinuous way for feeding Bobbins by the pallet, a feed structure is customized to load the pallet filled with Bobbins and unload the empty pallet once automatically at least. This structure can take place the exchange for pallets by manual, as a result time is saved and the efficiency of the assembly system is improved. The clamping structure for Bobbin consists of two sets of step motor and a ball screw so that can complete the action of clamping for144 locations in the pallet.

3) Mechanical design of assembly for Yoke and Bobbin

As the accomplishment of feed and clamping of Yokes and Bobbins, the assembly structure will make them get together. In the procedure of assembly, the Bobbin is inserted into the Yoke and the Yoke as the base of the Yoke-Bobbin (the structure of assembly of Yoke \& Bobbin). So the work table is designed to load the Yoke at first and then the Bobbin is inserted into it.

4) Mechanical design of feed for the Yoke-Bobbin

After the assembly of the Bobbin and the Yoke, the AirTAC HF Series Gripper is used for clamping the Yoke-Bobbin to the position for placing Bases, and the AirTAC STW Series Cylinder serves as the transmission actuator that transports the Yoke-Bobbin to the location of the Base. The clamping structure and transmission structure make up the feed structure for the Yoke-Bobbin.

5) Mechanical design of assembly for Base and Yoke-Bobbin

The assembly for the Base and the Yoke-Bobbin is the last step in this part of the assembly system. The feed for the Base is same as the Yoke that adopts the combination of vibration tray and linear feeder. Due to the different shape with Yokes, Bases are get separated by the simply dividing structure in the linear feeder without a customized structure for separating. And the location structure takes advantage of biaxial jaw to realize functions of clamping and location for the Base. In the procedure of assembly, the Yoke-Bobbin is inserted into the Base and the Base as the base of the first part assembly.

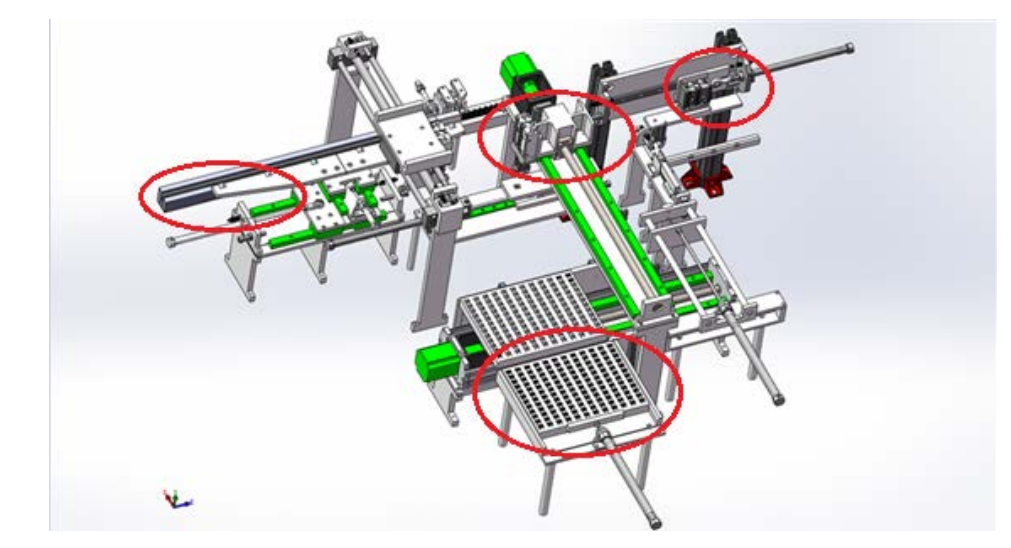

Figure 3. Diagram of Yoke, Bobbin and Base assembly part design. 
The mechanical design of each sub-structure can be shown in the Figure 4. Each sub-structure can be described as follows. 1) The structure of location for Yoke; 2) The structure of clamping for Yoke; 3) The structure of feed for Bobbin; 4) The structure of clamping for Bobbin; 5) The work table of assembly for Yoke and Bobbin; 6) The structure of clamping for Yoke-Bobbin; 7) The structure of feed for Base; 8) The structure of assembly for Base and Yoke-Bobbin (The no. of each sub-structure is corresponded to the no. as shown in Figure 4).

\section{Kinematic Simulation of Automated Assembly System for Relay}

After the three-dimensional model of the automated assembly system for relay is built. Kinematic simulation and analysis of the automated assembly system mainly focus on feasibility and validity of the mechanical design is implemented based on Solid Works Motion. In this section, we also choose the Yoke, Bobbin and Base assembly part to simulation and analysis.

\subsection{Kinematic Simulation of Yoke, Bobbin and Base Assembly Part Design}

1) Working process of Yoke, Bobbin and Base assembly part

In this Kinematic simulation and analysis, the event based motion is chosen as a solution paradigm [7]. In an event based motion, the motion of the assembly triggers the external action. And every event in the event based motion is corresponded with every mechanical action in the working process of assembly system. Based on the working process, the SFC (Sequential Function Chart) for PLC (Programmable Logic Controller) can be projected in related practical assembly system [8].

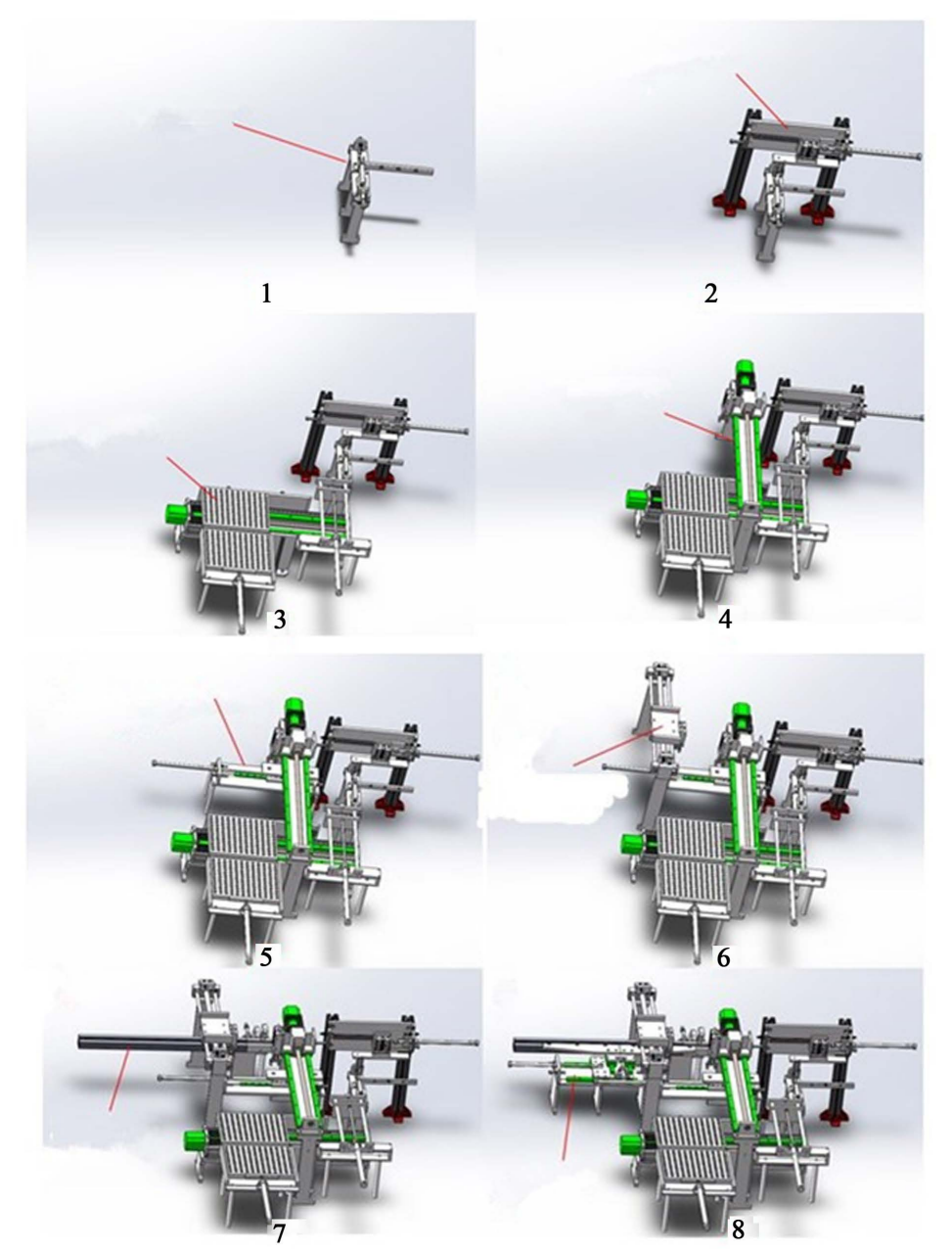

Figure 4. Diagram of each sub-structure design of Yoke, Bobbin and Base assembly part. 
Analyzing the working process of the Yoke, Bobbin and Base assembly part, the SFC is exported easily which can be shown in the Figure 5. And it's clear to recognize the every task (event) in the event based motion of this kinematic simulation and analysis.

2) Motion analysis of Yoke, Bobbin and Base assembly part design

The steps for motion analysis of the Yoke, Bobbin and Base assembly part can be described as follows. Firstly, to get the assembly of this part applied to motion analysis, the overall assembly is disbanded into each sub-assembly layer by layer in the window of the model. Next, extra relationships of sub-assemblies are added in Solid Works and the immovable components are fixed. Then motion motors which include linear motor and rotation motor are added to the structure which is necessary to realize the mechanical action in the window of motion paradigm. Finally, every task for the whole part is added in the window of event and task at the base of the SFC for PLC of this assembly part.

\subsection{Kinematic Simulation}

Table 1 is parts of the task table of working process for Yoke, Bobbin and Base assembly part. This table is generated automatically by Solid Works after the motion analysis of the mechanical design.

According to the table, the function of each mechanical action which each task represents for and the sequence of every task has been shown. And it's obvious that how far mechanical structure has traveled and how much time has been consumed by each task in the working process of whole assembly part in the table.

The simulated animation of working process for Yoke, Bobbin and Base assembly part can get from the motion analysis. The animation displays every detail of working process for the assembly part totally. And the interference between the adjoining structures is easily to observe in the animation which shows the relationships among mechanical structures.

Parts of animation at different time of working process for the assembly part is shown in Figure 6, verifying that the Yoke, Bobbin and Base assembly part can work properly and the mechanical structure don't interfere with each other during the operation.

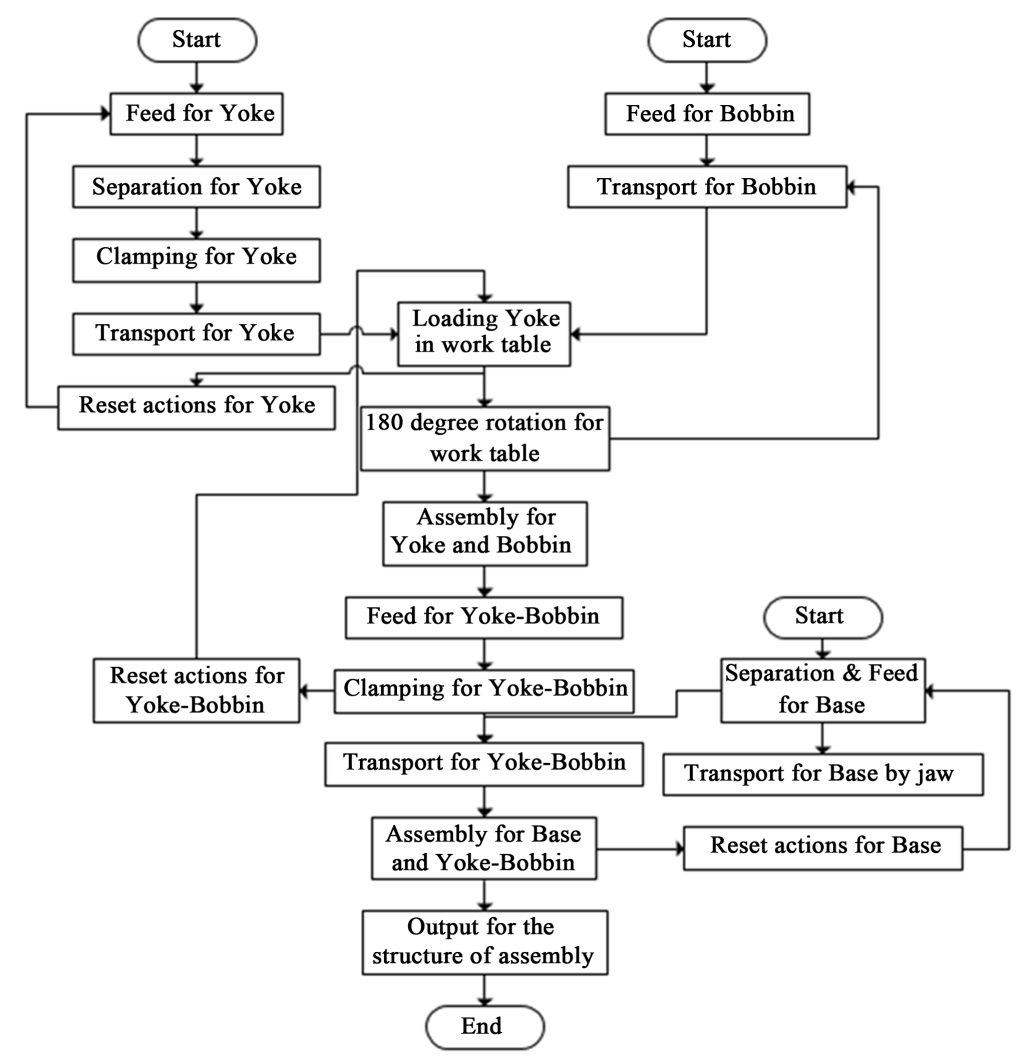

Figure 5. The SFC for PLC of Yoke, Bobbin and Base assembly part. 


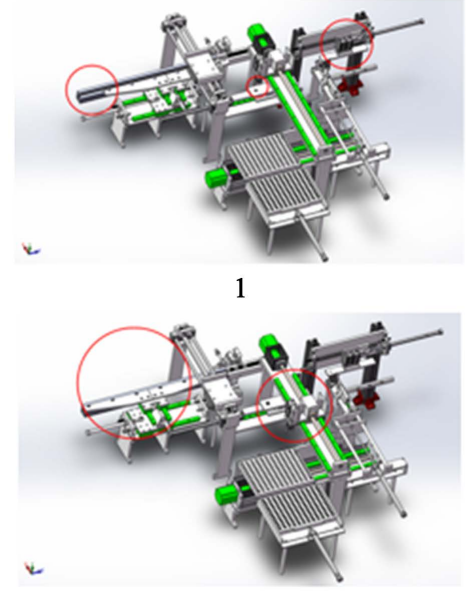

3

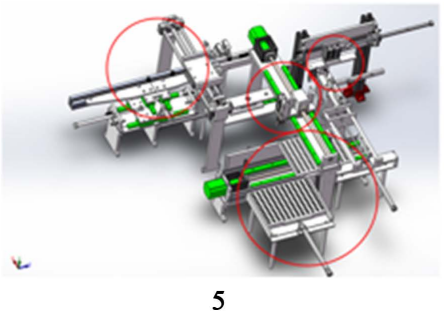

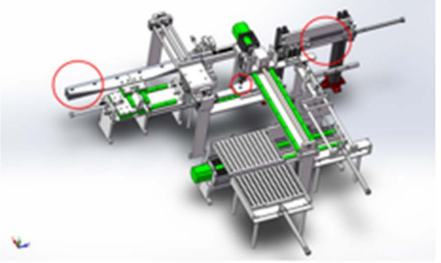

2

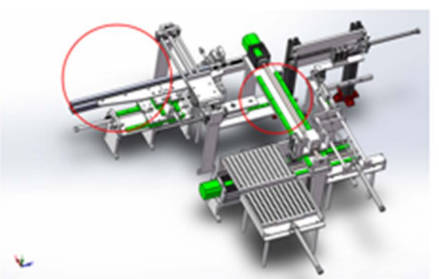

4

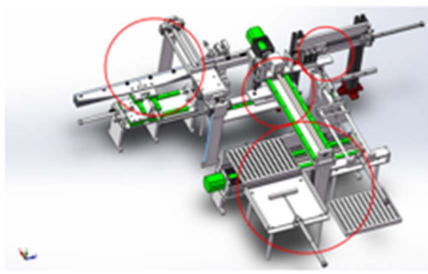

6

Figure 6. Screenshots of simulated animation at different time of the working process. 1 . at $0 \mathrm{~s} ; 2$. at $5 \mathrm{~s} ; 3$. at $10 \mathrm{~s} ; 4$. at $20 \mathrm{~s}$; 5. at $30 \mathrm{~s} ; 6$. at $40 \mathrm{~s}$ (Observe the change in the same cycle carefully).

Table 1. A part of task table of working process for Yoke, Bobbin and Base assembly part.

\begin{tabular}{|c|c|c|c|c|c|c|}
\hline \multirow{2}{*}{ Task } & \multirow{2}{*}{ Trigger } & \multirow{2}{*}{ Characteristic } & \multirow{2}{*}{ Parameter } & \multicolumn{3}{|c|}{ Time } \\
\hline & & & & Start (s) & End (s) & Duration (s) \\
\hline NO.169 Task & NO.168 Task & Base_3Z & $36.31(\mathrm{~mm})$ & 25.5 & 26 & 0.5 \\
\hline NO.170 Task & NO.169 Task & Location of Pallet & $20(\mathrm{~mm})$ & 26 & 26.5 & 0.5 \\
\hline NO.171 Task & NO.170 Task & Location of Base & $-10(\mathrm{~mm})$ & 26.5 & 27 & 0.5 \\
\hline \multicolumn{7}{|c|}{$\cdots$} \\
\hline NO.181 Task & NO.180 Task & Base_4Z & $35.49(\mathrm{~mm})$ & 26 & 28 & 0.5 \\
\hline NO.182 Task & NO.162 Task & Pallet for Yoke & $-150(\mathrm{~mm})$ & 26 & 28 & 2 \\
\hline NO.183 Task & NO.162 Task & Pallet for Yoke & -180 (deg) & 26 & 28 & 2 \\
\hline \multicolumn{7}{|c|}{$\ldots$} \\
\hline NO.193 Task & NO.191 Task & Bobbin_5 Y & $55.7(\mathrm{~mm})$ & 31.5 & 32 & 0.5 \\
\hline NO.194 Task & NO.191 Task & Up and down clamping for Bobbin & $-55.7(\mathrm{~mm})$ & 31.5 & 32 & 0.5 \\
\hline NO.195 Task & NO.194 Task & Right and left clamping for Bobbin & $-388.7(\mathrm{~mm})$ & 32 & 34 & 2 \\
\hline & & $\ldots$ & & & & \\
\hline
\end{tabular}

\subsection{Productivity Estimation for Yoke, Bobbin and Base Assembly Part}

The pneumatic equipment usually works at the pressure of 0.4 MPa to 0.6 MPa [9]. Because of the low load for driving of every actuator, the time of most cylinders that achieve a single motion is close to others in practice. The productivity estimation for the Yoke, Bobbin and Base assembly part can be calculated as 


$$
\begin{gathered}
(0.5+1) \times 2 \times 1.3 \div 2=1.95 s \approx 2 s \\
(0.5+1) \times 2 \times 1.3 \div 2.5=1.56 s \approx 1.6 s
\end{gathered}
$$

where, 0.5 represents the simulation time of assembly for Yoke and Bobbin. And 1 represents the simulation time of assembly for Yoke \& Bobbin and Base, both provided by the motion analysis of Solid Works. 2 stands for the assembly times of whole part assembly process for once. 1.3 is the coefficient of correction. Equation (1) and Equation (2) are corresponded to different amplification coefficients of simulation time in the whole motion analysis, which are 2 and 2.5 [10].

To sum up, on the stable work condition of this assembly part, about $1.6 \mathrm{~s}$ to $2 \mathrm{~s}$ is consumed to finish the per assembly of Yoke, Bobbin and Base. The productivity is in line with the actual requirements of enterprise.

\section{Conclusion}

In this paper, mechanical design and kinematic simulation are researched. Principle and mechanical structures of the automated assembly system for relay are presented. The mechanical design of the Yoke, Bobbin and Base assembly part is performed especially, mechanical design of feed, location, clamping and assembly for the Yoke, Bobbin and Base are included. Kinematic simulation and analysis of the Yoke, Bobbin and Base assembly part focused on working process in practical assembly are performed based on Solid Works Motion. The simulation results verify the validity of the mechanical design of the Yoke, Bobbin and Base assembly part. This paper provides an effective and reliable mechanical design for the automated assembly system for relay.

\section{Acknowledgements}

The authors would like to thank the 2015 Teaching Research Project of Shenzhen University under the code 00002409260803 and Churod Electronics Co., LTD in Dongguan, Guangdong, especially the help and advice have provided by the mechanical engineers and electrical engineers.

\section{References}

[1] De Fazio, T.L., Edsall, A.C. and Gustavson, R.E. (1991) A Prototype of Feature-Based Design for Assembly. Springer, Berlin Heidelberg, 369-392. http://dx.doi.org/10.1007/BFb0014287

[2] Wang, L., Keshavarzmanesh, S., Feng, H.-Y. and Buchal, R.O. (2009) Assembly Process Planning and Its Future in Collaborative Manufacturing: A Review. International Journal of Advanced Manufacturing Technology, 41, 132-144. http://dx.doi.org/10.1007/s00170-008-1458-9

[3] Bi, Z., Wang, L. and Lang, S. (2007) Current Status of Reconfigurable Assembly Systems. International Journal of Manufacturing Research, 2, 303-328. http://dx.doi.org/10.1504/IJMR.2007.014727

[4] Boer, C.R., Pedrazzoli, P., Sacco, M., Rinaldi, R., De Pascale, G. and Avai, A. (2001) Integrated Computer Aided Design for Assembly Systems. CIRP Annals, 50, 17-20. http://dx.doi.org/10.1016/S0007-8506(07)62061-7

[5] Zhu, W., Wang, C., Wu, D., Tan, H. and Yang, F. (2009) Branch Aircraft’s Digital Assembly Process Design and Simulation. IET International Communication Conference on Wireless Mobile and Computing, Shanghai, 7-9 December 2009, 413-416.

[6] http://www.airtac.com/en/pro.aspx?c kind=4\&c kind2=19\&c kind3=41\&c kind4=54/

[7] http://www.solidworks.com/sw/products/simulation/motion-analysis.html

[8] Yu, J., Wang, C., Yu, H. and Zhang, W. (2009) Generation of Optimized Assembly Sequences Based on Priority Rules Screening. Applied Mechanics and Materials, 16, 130-134. http://dx.doi.org/10.4028/www.scientific.net/AMM.16-19.130

[9] Xu, L.D., Wang, C.G., Bi, Z.M. and Yu, J.P. (2012) Auto Assem: An Automated Assembly Planning System for Complex Products. IEEE Transactions on Industrial Informatics, 8, 1551-1561. http://dx.doi.org/10.1109/TII.2012.2188901

[10] Jia, C.H., Liu, Y.B. and Xia, X.T. (2009) Research and Application of Digital Assembly Process Planning and Simulative Validation. International Conference on Mechatronics and Automation, Changchun, 9-12 August 2009, 22032207.

http://ieeexplore.ieee.org/xpl/login.jsp?tp=\&arnumber=5246737\&tag=1\&url=http\%3A\%2F\%2Fieeexplore.ieee.org\%2 Fxpls\%2Fabs_all.jsparnumber\%3D5246737\%26tag\%3D1 